\title{
POLARIMETRIC CALIBRATION OF L-BAND AIRBORNE SAR DATA
}

\author{
A. Maiti ${ }^{1}$, S. Kumar ${ }^{2}$, V. Tolpekin ${ }^{3}$, S. Agarwal ${ }^{2} *$ \\ ${ }^{1}$ Faculty of Geo-Information Science and Earth Observation, University of Twente - a.maiti@utwente.nl \\ ${ }^{2}$ Indian Institute of Remote Sensing, ISRO - (shashi, shefali_a)@iirs.gov.in \\ ${ }^{3}$ ICEYE, Espoo, Finland - valentyn.tolpekin@iceye.fi
}

Commission III, WG III/2

KEY WORDS: PolSAR, radiometric calibration, phase calibration, crosstalk, channel imbalance

\begin{abstract}
:
The PolSAR calibration ensures that the relationship between the SAR observations and the target characteristics on the ground are consistent and resembles the theoretical estimation which in turn improves the overall data quality. Essentially, calibration prevents the propagation of uncertainty into further analysis to characterise the target. In this study, the UAVSAR L-Band data of Rosamond dry lake bed has been calibrated. The calibration of amplitude and phase are carried out with the help of the corner reflector array present in the Rosamond site. The dataset is further calibrated for the crosstalk and channel imbalance using the Quegan's distortion model. Since the crosstalk distortion model requires an accurate estimation of the covariance matrix, the optimal kernel size for the its computation is selected based on the distortion model behaviour with varying window sizes. Furthermore, the effectiveness of the calibration process has been studied using polarimetric signatures and other statistical measures.
\end{abstract}

\section{INTRODUCTION}

The polarimetric synthetic aperture radar (PolSAR) data represent important geophysical properties of the target scene. However, the radar measurements have to be consistent and quantifiable with sufficient accuracy and precision in order to quantitatively analyse these properties. In practice, the radar measurements can be attenuated by several internal and external factors such as improper channel isolation, antenna pattern, topographical variations, system noise etc. These factors severely influence the quality of PolSAR data and are hardly avoidable. Evidently, appropriate corrections are necessary in order to make reliable measurements from PolSAR data (Freeman, 1989). In this regard, the PolSAR calibration improves the overall data quality by ensuring that the radar measurements are quantifiable, reproducible and accurately reflect the target properties.

The calibration approach of SAR data may vary depending upon the sensor, frequency of the signal, acquisition mode and the platform. Among the available acquisition modes, full polarimetric or quadpol SAR data is widely popular as it contains more information about the target compared to the data acquired in other modes. In case of quadpol dataset, ideally the ratio of the copolarised (copol) channel measurements should remain constant. Similarly, according to the principle of reflection symmetry, the measurements in the crosspolarised (crosspol) channel ideally should be identical. Also, the uncalibrated POLSAR data suffers from channel imbalance (Noora, 2014). Moreover, improper channel isolation causes distortions known as crosstalk (Zyl, Kim, 2011). Furthermore, irregular pixel spacing in SAR data leads to the error in estimation of Radar Cross Section (RCS) (Freeman et al., 1992). The effect of these anomalies can be observed as the irregularities in the scattering pattern and the distortion of the polarimetric signatures of the known targets.

\footnotetext{
* A. Maiti
}

PolSAR calibration is a complex process which involves estimation of different calibration parameters by statistically comparing the uncalibrated data with the ideal theoretical models. In this regard, radiometric calibration and phase calibration plays a pivotal role by rectifying the effect of irregular antenna pattern and phase ambiguities. Evidently, after appropriate radiometric and phase calibration, the remaining distortions due to crosstalk (Jackson, Lee-Elkin, 2019) and channel imbalance (Shi et al., 2019) can be modelled and rectified accordingly (Fore et al., 2015). In this context, two well established algorithms to minimise the crosstalk and channel imbalance have been developed by Quegan (Quegan, 1994) and Ainsworth (Ainsworth et al., 2006). The Ainsworth's algorithm adopts an iterative approach to estimate the crosstalk and channel imbalance parameters and enforces weaker constraints such as scattering reciprocity on the dataset (Ainsworth et al., 2006). In contrast, the Quegan's algorithm adopts a simpler model to estimate the crosstalk parameters. However, it enforces stricter assumptions on the dataset such as azimuthal symmetry and also ignores helicity in the backscattering (Quegan, 1994).

The main objective of this study is to calibrate the PolSAR data and observe its effectiveness by statistically comparing the uncalibrated and calibrated data and synthesising the corresponding polarimetric signatures. Here, the polarimetric calibration has been performed on the NASA UAVSAR L-Band dataset.

\section{METHODOLOGY}

The work-flow adopted in this research is graphically depicted in fig. 1. At first, a subset operation has been applied in order to select a suitable area of adequate size as shown in the fig. 2 . After that all the corner reflectors in the selected scene has been identified and their RCS values has been theoretically estimated. These RCS values have been used to further calculate the absolute radiometric calibration parameters at the 
peak of each oversampled corner reflector point. In addition, the copol channel imbalance parameter and HH-VV phase error have also been estimated for each corner reflector. Since the absolute calibration parameter is a function of incidence angle (Fore et al., 2015, eq. (7)), an appropriate polynomial fit of the absolute calibration parameters has been performed with respect to the normalised incidence angle. Similarly, copol phase bias is expressed as a polynomial function of the normalised incidence angle. On the other hand, crosspol channel imbalance parameter has been estimated from the ratio of the mean crosspol backscattering powers from a relatively large homogeneous area. The cross polarisation phase bias is estimated from the same homogeneous area as the mean phase error between two crosspol channels. These parameters has been further used to perform the radiometric and phase calibration of the dataset.

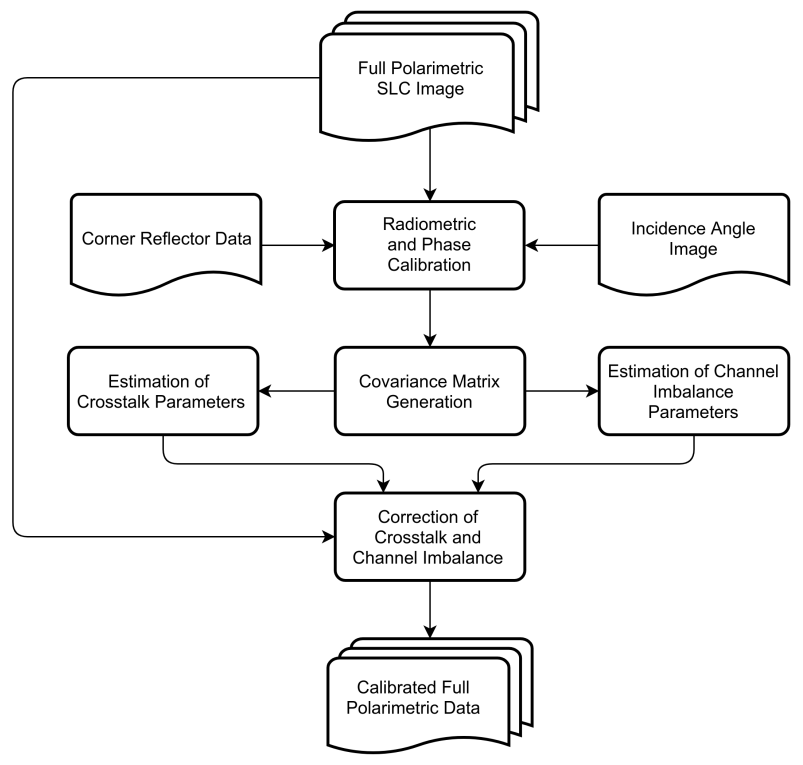

Figure 1. The research work-flow

The relation between observed scattering matrix $\left(O=\left[O_{i, j}\right]\right.$ : $i, j \in\{H, V\})$ and the actual scattering matrix $\left(S=\left[S_{i, j}\right]\right.$ : $i, j \in\{H, V\})^{1}$ can be expressed as in equations (1) (Fore et al., 2015)

$$
\begin{aligned}
& \mathbf{S}_{o}^{\prime}=Y \mathbf{D} \mathbf{S}_{\tau}^{\prime}+\mathbf{N} \\
& \mathbf{S}_{o}^{\prime}=\left[\begin{array}{llll}
s_{h h}^{\prime} & s_{v h}^{\prime} & s_{h v}^{\prime} & s_{v v}^{\prime}
\end{array}\right]^{\mathrm{T}} \\
& \mathbf{D}=\left[\begin{array}{cccc}
1 & w & v & v w \\
u & 1 & u v & v \\
z & w z & 1 & w \\
u z & z & u & 1
\end{array}\right]\left[\begin{array}{cccc}
\alpha k^{2} & 0 & 0 & 0 \\
0 & \alpha k & 0 & 0 \\
0 & 0 & k & 0 \\
0 & 0 & 0 & 1
\end{array}\right] \\
& \mathbf{S}_{\tau}^{\prime}=\left[\begin{array}{llll}
s_{h h} & s_{v h} & s_{h v} & s_{v v}
\end{array}\right]^{\mathrm{T}} \\
& \mathbf{N}=\left[\begin{array}{llll}
n_{h h} & n_{v h} & n_{h v} & n_{v v}
\end{array}\right]^{\mathrm{T}}
\end{aligned}
$$

where, $u, v, w$ and $z$ are the crosstalk parameters having complex values. Here, $k$ and $\alpha$ are the copol and crosspol channel imbalance terms respectively and $Y$ is the absolute gain parameter. The system noise $\left(\mathbf{N}=\left[n_{i, j}\right]: i, j \in\{H, V\}\right)$ is ignored for this study. Since the radiometric and phase calibrations have already been performed, it can be assumed

\footnotetext{
$1 i, j$ are respectively transmit and receive polarisation channels and $\mathrm{H}$ $\mathrm{V}$ signify the horizontal and vertical polarisations respectively.
}

that equations in (2) are satisfied (Fore et al., 2015).

$$
\begin{gathered}
Y=1 \\
k=\frac{1}{\sqrt{\alpha}}
\end{gathered}
$$

The covariance matrix computed using equation (3). The window size used to compute the covariance matrix, plays an important role in the estimation of the crosstalk parameters. In this study, crosstalk estimation has been performed using different window size and the window size of $7 \times 7$ found to be optimal.

$$
\mathbf{C}=\left\langle\mathbf{S S}^{\dagger}\right\rangle
$$

Here, the crosstalk has been modelled using the Quegan's method. Quegan's crosstalk modelling assumes the following conditions are satisfied:

1. The acquired dataset is fully polarimetric and available in the form of the scattering matrix.

2. The observed scattering matrix can be modelled as a linear system.

3. Scattering reciprocity is satisfied unless the target is physically altered.

4. In the case of distributed targets, cross-polarised channels are not correlated.

5. The off-diagonal terms of the distortion matrix are small compared to the diagonal terms.

Consequently, according to Quegan's assumptions, the true covariance matrix has the form as shown in (4) (Quegan, 1994).

$$
\mathbf{C}_{\mathbf{Q}}=\left[\begin{array}{cccc}
\sigma_{h h h h} & 0 & 0 & \sigma_{h h v v} \\
0 & \beta & \beta & 0 \\
0 & \beta & \beta & 0 \\
\sigma_{h h v v}^{*} & 0 & 0 & \sigma_{v v v v}
\end{array}\right]
$$

According to eq. (1), the Quegan's distortion model assumes that the correlation between crosspol channels is zero. Therefore the correlation between crosspol channels has been computed using the same $7 \times 7$ sliding window. The crosstalk parameters are only estimated only from the points where the correlation is less than 0.3 . Following this, the crosstalk parameters are estimated using the equations in (5) (Quegan, 1994).

$$
\begin{aligned}
u & =\frac{\left(C_{44} C_{21}-C_{41} C_{24}\right)}{\Delta} \\
v & =\frac{\left(C_{11} C_{24}-C_{21} C_{14}\right)}{\Delta} \\
w & =\frac{\left(C_{44} C_{31}-C_{41} C_{34}\right)}{\Delta} \\
z & =\frac{\left(C_{11} C_{34}-C_{31} C_{14}\right)}{\Delta} \\
\Delta & =C_{11} C_{44}-\left|C_{14}\right|^{2}
\end{aligned}
$$

Accordingly, the channel imbalance has been derived using the equations (6) (Fore et al., 2015). In equation (6e), Quegan 
assumed $m=1$, however, the parameter $m$ can be better estimated using equation (6f) (Kimura et al., 2004).

$$
\begin{gathered}
\alpha=\frac{\left|\alpha_{1} \alpha_{2}\right|-m^{2}+\sqrt{\left(\left|\alpha_{1} \alpha_{2}\right|-m\right)^{2}+4 m\left|\alpha_{2}\right|^{2}}}{2\left|\alpha_{2}\right|} \frac{\alpha_{1}}{\left|\alpha_{1}\right|} \\
\alpha_{1}=\frac{\left(C_{22}-u C_{12}-v C_{42}\right)}{X} \\
\alpha_{2}=\frac{X^{*}}{\left(C_{33}-z^{*} C_{31}-w^{*} C_{34}\right)} \\
X=C_{32}-z C_{12}-w C_{42} \\
m=\frac{n_{v h}}{n_{h v}} \\
m \approx \frac{\left\langle s_{v h}^{\prime} s_{v h}^{\prime *}\right\rangle}{\left\langle s_{h v}^{\prime} s_{h v}^{\prime *}\right\rangle}
\end{gathered}
$$

Finally, the crosstalk and channel imabalance have been corrected using the equation (7a) which has been directly derived from equation (1a).

$$
\begin{gathered}
\mathbf{S}_{\tau}^{\prime}=\mathbf{D}^{-1} \mathbf{S}_{o}^{\prime} \\
\mathbf{D}^{-1}=\left(\begin{array}{cccc}
1 & -w & -v & v w \\
-u / \sqrt{\alpha} & 1 / \sqrt{\alpha} & u v / \sqrt{\alpha} & -v / \sqrt{\alpha} \\
-z \sqrt{\alpha} & w z \sqrt{\alpha} & \sqrt{\alpha} & -w \sqrt{\alpha} \\
w z & -z & -u & 1
\end{array}\right)
\end{gathered}
$$

The numerical inversion of the calibration matrix can be avoided using the analytical solution presented in equation (7b) (Fore et al., 2015). The effect of polarisation has been observed using the polarimetric signatures of the oversampled corner reflector pixels. Additionally the variations among the copol channels and the crosspol channels has been shown using the respective scatter plots.

There are several metrics to measure the effects of the calibration on PolSAR data. Some of these metrics are extremely useful for analysing the data quality and estimating the residual noise and uncertainty. The metrics used in this study to evaluate the calibration is discussed below. Any change in the residual noise in the data should be reflected in the crosstalk and channel imbalance parameters estimated before and after performing calibration respectively. Moreover, according to (Wang et al., 2011), Maximum Normalised Error (MNE) is a generalised metric to evaluate the proportion of noise present in the data. Furthermore, (Villano, Papathanassiou, 2013) suggested that the SNR of cross-pol channels of SAR data is crucial since the powers of the backscattered signal are comparatively very low with respect to backscatter powers of the co-pol channels. Additionally, the polarimetric signatures of the known targets such as corner reflectors can reveal the quality of PolSAR data when compared with the ideal theoretical response (Cloude, 2009). Polarimetric signatures are particularly useful to evaluate the radiometric and phase calibration (Fore et al., 2015).

Previous studies suggest that, $-20 \mathrm{~dB}$ of MNE translates into $-26 \mathrm{~dB}$ of crosstalk and the recommended value of MNE is $25 \mathrm{~dB}$ (Wang et al., 2011). On the contrary, the recommended threshold for the crosstalk is $-35 \mathrm{~dB}$ (ESA, 2004). Therefore, the threshold for MNE has been set to $-30 \mathrm{~dB}$ for this study.

Here, all the processing have been performed using Python
(Rossum, 1995) and open source libraries such as scipy (Virtanen et al., 2020), gdal (GDAL Development Team, 2019).

\section{STUDY AREA AND DATA}

\subsection{Study Area}

Rosamond lake is situated in the Mojave desert in the state of California, USA. It is a natural dry lake bed having a large and flat surface with curvature of less than $\sim 40 \mathrm{~cm}$ over a distance of $9 \mathrm{kms}$. The surface of this region is very hard and void of any kind of vegetation. At the southern part of the lake bed array of corner reflectors having 38 trihedral corner reflectors of different size has been deployed and it is being used as permanent radar cal-val site by NASA. Around $10 \mathrm{~km}^{2}$ area at the southern part, containing the entire corner reflector array has been used as the study area.

\subsection{Dataset}

In this study, a fully polarimetric (Quad-pol) Single look Slant range Complex monostatic acquisition of UAVSAR LBand has been used as dataset. The acquisition date of the dataset is September 20, 2016. Along with this, the corner reflector data of the Rosamond array of the same date has been used to theoretically estimate the radar cross section (RCS) of the corner reflectors as the corner reflector data contains accurate information about the azimuth angle, tilt angle and the location of the corner reflectors. The corner reflectors present in the scene are demarcated in fig. 2. The corner reflectors oriented towards the radar look angle are marked with circle and coloured according to their size. The disoriented corner reflectors with respect to radar look angle are marked with square.

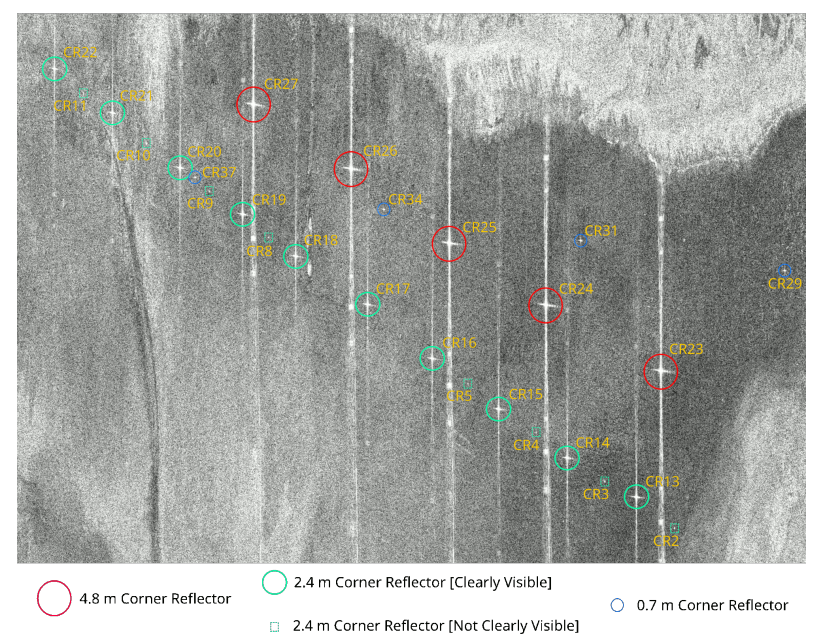

Figure 2. Intensity image of uncalibrated HH-band highlighting different corner reflectors

\section{RESULTS AND DISCUSSION}

\subsection{Polarimetric Signatures}

The polarimetric signature depicts normalised RCS power of a target as a function of ellipticity angle and the orientation angle of the polarisation ellipse. In case of trihedral corner reflector, the normalised RCS should remains unaffected by the orientation angle for a given ellipticity angle. For a given 
orientation angle, the RCS of trihedral corner reflector should attain the peak when ellipticity angle is $45^{\circ}$. Therefore the theoretical polarimetric signature of a trihedral corner has the shape as shown in (Zyl, Kim, 2011, Fig. 2-7). The copol polarimetric signatures before and after the calibration are shown in fig. 3. Here fig. 3(a) and fig. 3(b) are the polarimetric signatures of one $4.8 \mathrm{~m}$ trihedral corner reflector and one $2.4 \mathrm{~m}$ corner reflector respectively and the fig. 3(c), fig. 3(d) are the polarimetric signatures of the respective corner reflectors after the calibration has been performed. In fig. 3(a) and in fig. 3(b), the distortions in the polarimetric signature are clearly visible. However, after the calibration, nearly perfect polarimetric signature has been obtained. The HH-VV power imbalance for maximum $\mathrm{HH}$ was at $0.43 \mathrm{~dB}$ which has been modified to $-0.13 \mathrm{~dB}$ after the calibration for the $2.4 \mathrm{~m}$ corner reflector and the same has been changed from $0.34 \mathrm{~dB}$ to $0.21 \mathrm{~dB}$ for the $4.8 \mathrm{~m}$ corner reflector. The distortions in the crosspol signatures were negligible in the uncalibrated dataset which remained unchanged after performing the calibration.

\subsection{Variation in copol and crosspol Channels}

The effect of calibration can observed in the variation of copol channel and crosspol channels. Ideally, after the calibration the both copol backscattering values should lie near the $y=x$ line compared to the uncalibrated dataset. Same principle is applicable to the crosspol channels. In the fig. 4, the fig. 4(a) is scatterplot of the $\mathrm{HH}$ and $\mathrm{VV}$ before the calibration while the fig. 4(b) is the scatterplot of the same after the calibration. Similarly, the fig. 4(c) and the fig. 4(d) represents the scatterplot of $\mathrm{HV}$ and $\mathrm{VH}$ before and after calibration respectively.

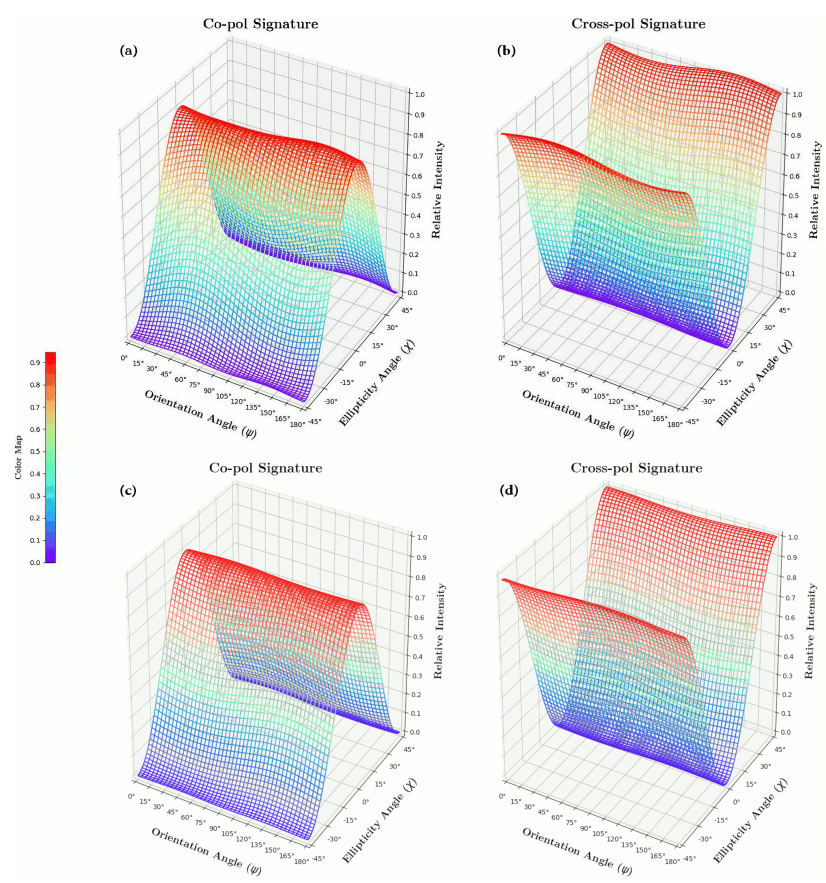

Figure 3. Polarimetric signatires of CR23. (a), (c) Copolar Polarimetric signatures before and after the calibrabtion respectively. (b), (d) Crosspolar Polarimetric signatures before and after the calibrabtion respectively

Evidently, the slope angle of the regression line has been improved to $44.82^{\circ}$ in fig. 4(b) from $44.11^{\circ}$ in fig. 4(a) wherein, the slope of the regression line has been improved to $33.879^{\circ}$ in fig. 4(d) from $23.89^{\circ}$ in fig. 4(c). Therefore, both copol and

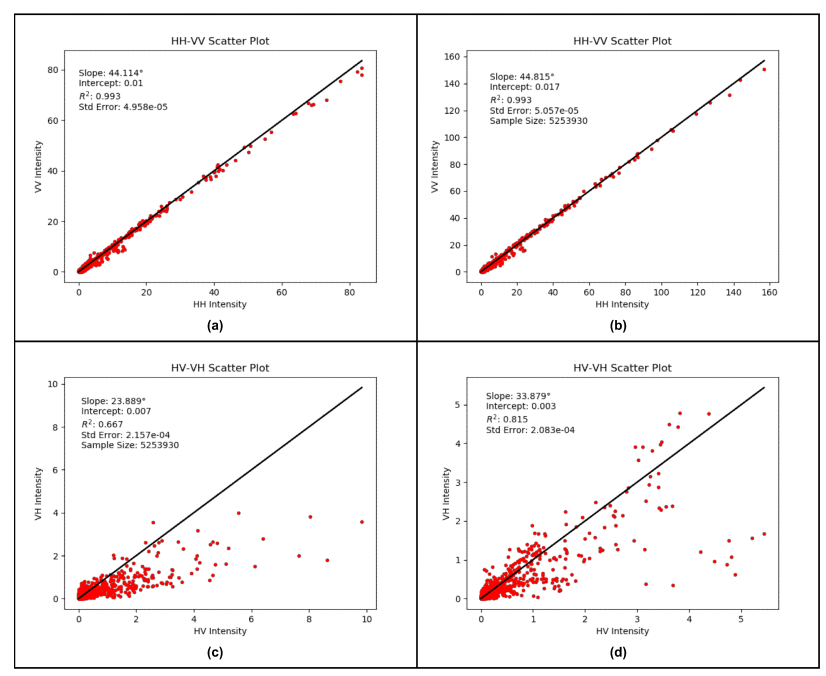

Figure 4. (a) Scatter Plot of HH and VV before calibration, (b) Scatter Plot of HH and VV after calibration, (c) Scatter Plot of $\mathrm{HV}$ and VH before calibration, (c) Scatter Plot of HV and VH after calibration

crosspol channels are more organised along the $y=x$ line after the calibration.

\subsection{Noise Estimation}

The variations of crosstalk parameters estimated using Quegan's algorithm have been observed before and after the crosstalk calibration. In Figure fig. 6, crosstalk parameters are plotted for the range direction over 2750 range pixels. It can be observed that the parameter $u$ varies roughly between $-28 \mathrm{~dB}$ to $-34 \mathrm{~dB}$ before the calibration and it varies roughly between $-34 \mathrm{~dB}$ to $-38 \mathrm{~dB}$ after the calibration. There are no anomalies in the estimation of $u$ as the residual of $u$ is less than the estimated $u$ before the calibration in all the cases. The parameter $v$ is estimated to be nearly $-20 \mathrm{~dB}$ and it does not show much variations with respect to the range direction before the calibration. However, residual of $v$ varies between $-35 \mathrm{~dB}$ and $-43 \mathrm{~dB}$ and exhibits a similar pattern to that of residual $u$. The parameter $w$ mostly varies around $-35 \mathrm{~dB}$ before the calibration and it is estimated to be roughly between $-35 \mathrm{~dB}$ $42 \mathrm{~dB}$ after the calibration. The parameter $z$ is estimated to be in the range of $-34 \mathrm{~dB}$ to $-36 \mathrm{~dB}$ before calibration and in the range of $-35 \mathrm{~dB}$ to $-39 \mathrm{~dB}$ after the calibration.

Accordingly, Figure fig. 5 shows the variation of MNE before performing the crosstalk calibration. The MNE before performing the crosstalk varied between $-18.41 \mathrm{~dB}$ and $21.63 \mathrm{~dB}$. However, MNE has been reduced to the range of $-26.69 \mathrm{~dB}$ to $-33.81 \mathrm{~dB}$ after crosstalk calibration using Quegan's algorithm. It is also evident from Figure fig. 5 that a large number of range pixels do not satisfy the threshold of $-30 \mathrm{~dB}$.

\section{CONCLUSION}

This study analysed the effect of the calibration on the PolSAR data. The comparative analysis of the dataset before and after calibration shows that the Quegan's algorithm is capable of modelling the crosstalk with acceptable accuracy. However, the proper radiometric and phase calibration is necessary before the estimation of crosstalk. It is also worth noting that, 

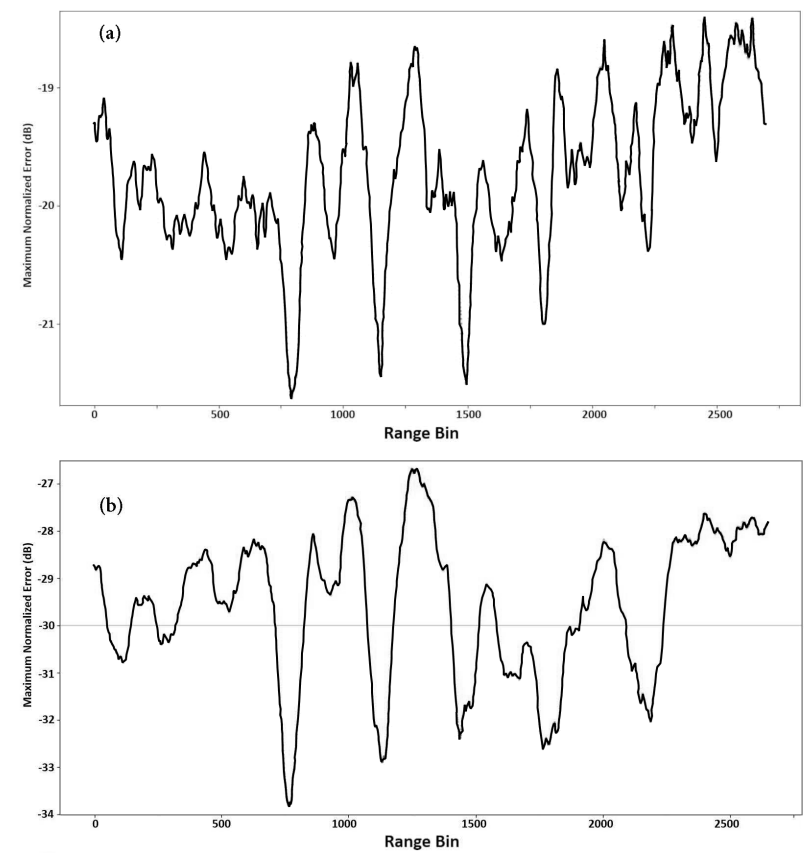

Figure 5. Variation of MNE with respect to the range direction (a) Before Calibration, (b) After Quegan's calibration.

the radiometric calibration is also important as it partially reduces the channel imbalance as well. Moreover, choice of window size in covariance matrix computation can influence the estimation of crosstalk parameters to a certain extent. Moreover, variations of MNE in this experment shows that Quegan's algorithm occasionaly fails to minimize it below the acceptable threshold (ESA, 2004).

In future work, it would be interesting to observe the performance of the Quegan's algortithm compared to the more advanced crosstalk models such as Ainsworth's algorithm. Also more in depth analysis can be performed by analysing the changes in the roll invariant parametric spaces and the changes in scattering pattern using different decompostion modelling.

\section{REFERENCES}

Ainsworth, T. L., Ferro-Famil, L., Lee, J. S., 2006. Orientation Angle Preserving a Posteriori Polarimetric SAR Calibration. IEEE Trans. Geosci. Remote Sens., 44(4), 9941003. http://ieeexplore.ieee.org/document/1610834/.

Cloude, S., 2009. Polarisation: Applications in Remote Sensing. First edn, Oxford University Press, Chippenham, Wiltshire.

ESA, 2004. Recommendation at CEOS CAL/VAL 2004.

Fore, A. G. et al., 2015. UAVSAR Polarimetric Calibration. IEEE Trans. Geosci. Remote Sens., 53(6), 3481-3491. http://ieeexplore.ieee.org/document/7012094/.

Freeman, A., 1989. The Need For Sar Calibration. 12th Can. Symp. Remote Sens. Geosci. Remote Sens. Symp., 1, IEEE, 230-233.

Freeman, A., Zyl, J., Klein, J. D., Zebker, H. A., Shen, Y., 1992. Calibration of Stokes and scattering matrix format polarimetric SAR data. IEEE Trans. Geosci. Remote Sens., 30(3), 531-539.
GDAL Development Team, 2019. GDAL - Geospatial Data Abstraction Library, Version 3.0.2. Open Source Geospatial Foundation.

Jackson, J. A., Lee-Elkin, F., 2019. Exploiting Channel Crosstalk for Polarimetric SAR Compressive Sensing. IEEE Transactions on Aerospace and Electronic Systems, 1-1.

Kimura, H., Mizuno, T., Papathanassiou, K. P., Hajnsek, I., 2004. Improvement of Polarimetric SAR Calibration based on the Quegan Algorithm. IEEE Int. IEEE Int. IEEE Int. Geosci. Remote Sens. Symp. 2004. IGARSS '04. Proceedings. 2004, 1number C, IEEE, 184-187.

Noora, A., 2014. Polarimetric Cross-Talk in SAR System Induced by Antenna Cross- Pol Pattern. Eur. Conf. Synth. Aperture Radar, 1365-1368.

Quegan, S., 1994. A Unified Algorithm for Phase and Cross-talk Calibration of Polarimetric Data - Theory and Observations. IEEE Trans. Geosci. Remote Sens., 32(1), 89-99.

Rossum, G., 1995. Python reference manual. Technical report, NLD.

Shi, L., Li, P., Yang, J., Zhang, L., Ding, X., Zhao, L., 2019. Co-polarization channel imbalance phase estimation by cornerreflector-like targets. ISPRS Journal of Photogrammetry and Remote Sensing, 147, 255 - 266.

Villano, M., Papathanassiou, K. P., 2013. SNR and Noise Variance Estimation in Polarimetric SAR data. ESA PolInSAR Workshop, ESA, Frascati, Italy.

Virtanen, P., Gommers, R., Oliphant, T. E., Haberland, M., Reddy, T., Cournapeau, D., Burovski, E., Peterson, P., Weckesser, W., Bright, J., van der Walt, S. J., Brett, M., Wilson, J., Jarrod Millman, K., Mayorov, N., Nelson, A. R. J., Jones, E., Kern, R., Larson, E., Carey, C., Polat, İ., Feng, Y., Moore, E. W., Vand erPlas, J., Laxalde, D., Perktold, J., Cimrman, R., Henriksen, I., Quintero, E. A., Harris, C. R., Archibald, A. M., Ribeiro, A. H., Pedregosa, F., van Mulbregt, P., Contributors, S. . ., 2020. SciPy 1.0: Fundamental Algorithms for Scientific Computing in Python. Nature Methods.

Wang, Y., Ainsworth, T. L., Lee, J. S., 2011. Assessment of System Polarization Quality for Polarimetric SAR Imagery and Target Decomposition. IEEE Trans. Geosci. Remote Sens., 49(5), 1755-1771. http://ieeexplore.ieee.org/document/5661826/.

Zyl, J., Kim, Y., 2011. Synthetic Aperture Radar Polarimetry. John Wiley \& Sons, Inc., Hoboken, NJ, USA. 

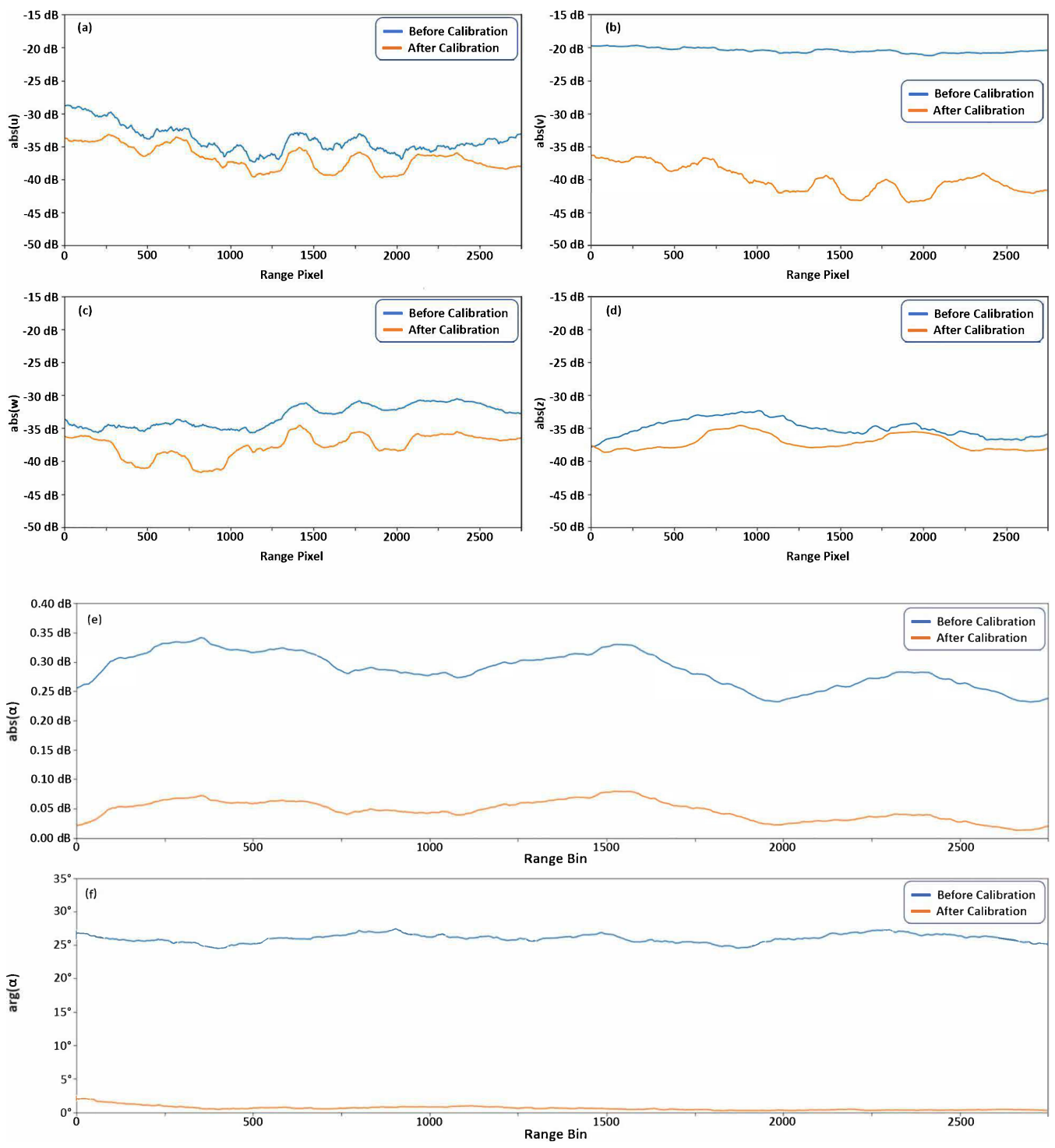

Figure 6. Variation of crosstalk and channel imbalance parameters estimated using Quegan's algorithm. The subfigures shows the variation of the parameters as follows: (a) $\operatorname{abs}(u),(\mathrm{b}) \operatorname{abs}(v),(\mathrm{c}) \operatorname{abs}(w),(\mathrm{d}) \operatorname{abs}(z),(\mathrm{e}) \operatorname{abs}(\alpha),(\mathrm{f}) \arg (\alpha) \operatorname{All}$ the crosstalk parameters and the channel imbalance parameter have reduced after performing calibration. 